\title{
Cambiando el paradigma educativo: propuesta de una estrategia educativa para la enseñanza-aprendizaje en cirugía vascular. Prueba piloto
}

\author{
Luis Fernando García Valenciaa
}

\begin{abstract}
Resumen: La preocupación por los escasos conocimientos en temas relacionados con la patología vascular en numerosos médicos generales ha llevado a cuestionar si el método tradicional de enseñanza en el área de cirugía vascular y angiología de la Universidad Militar Nueva Granada (UMNG) es el más apropiado y práctico para construir un conocimiento duradero, que pueda ser aplicado. En este trabajo analizamos los resultados de la implementación de tecnologías de la información y la comunicación (TIC) en la enseñanza-aprendizaje de los conocimientos que el médico general debe tener en cuanto a patologías vasculares, particularmente, las más frecuentes en la práctica clínica. Se trató de un modelo de aula virtual con módulos en que se presentaron los temas del currículo actual de la materia Patologías Vasculares y Angiología del Programa de Medicina de la UMNG, siguiendo el modelo de aula invertida (flipped classroom). Entre los resultados destaca una mejoría significativa de las notas en los estudiantes evaluados luego de que cursaron le módulo virtual, tanto en los casos en que ya habían llevado a cabo la rotación en el servicio de Cirugía Vascular, como en los que no lo habían hecho. La evaluación de esta prueba piloto se hizo con miras a determinar la efectividad de implementar en el futuro un módulo virtual aplicable a mayor escala en la formación básica del médico general en la Facultad de Medicina de la UMNG.
\end{abstract}

Palabras clave: aprendizaje; construcción del conocimiento; enseñanza; flipped classroom; módulo virtual de aprendizaje; tecnologías de la información y la comunicación; patología vascular

Fecha de recepción: 6 de noviembre de 2018 Fecha de aprobación: 23 de julio de 2019

Cómo citar: García Valencia LF. Cambiando el paradigma educativo: propuesta de una estrategia educativa para la enseñanza-aprendizaje en cirugía vascular. Prueba piloto. Revista Med. 27(2): 37-47. Disponible en: https://revistas.unimilitar.edu.co/index.php/rmed/article/view/4845

a Especialista en Cirugía Vascular y Angiología, Especialista en Docencia Universitaria. Hospital Militar Central, Bogotá DC, Colombia. Correo electrónico: fegava1@me.com 


\title{
Changing the Educational Paradigm: Educational Strategy Proposal for Vascular Surgery Teaching-Learning. Pilot Test
}

\begin{abstract}
The concern about numerous general practitioners' scarce knowledge of vascular pathology has led to questioning whether the traditional teaching method in the field of vascular surgery and angiology at the Universidad Militar Nueva Granada (UMNG) is the most appropriate and practical to build lasting and applicable knowledge. This paper discusses the results of implementing information and communication technologies (ICT) in the teaching-learning of knowledge of vascular pathologies, particularly the most frequent ones in clinical practice, that the general practitioner should have. It is a virtual classroom model with modules that present the current curriculum topics of the Vascular Pathologies and Angiology subject of the UMNG Medicine Program, following the flipped classroom model. The results include significant improvement in the marks of the students evaluated after completing the virtual module, whether they had already done a rotation in the Vascular Surgery service or not. The assessment of this pilot test was carried out to determine the effectiveness of implementing a virtual module applicable on a larger scale in the future to the basic training of general practitioners at the UMNG School of Medicine.
\end{abstract}

Keywords: Learning; knowledge building; teaching; flipped classroom; virtual learning module; information and communications technology; vascular pathology

\section{Transformando o paradigma educativo: proposta de uma estratégia educativa para o ensino-aprendizagem em cirurgia vascular. Teste-piloto}

Resumo: A preocupação pelos escassos conhecimentos em temas relacionados com a patologia vascular em inúmeros clínicos gerais tem levado a questionar se o método tradicional de ensino na área de cirurgia vascular e angiologia da Universidad Militar Nueva Granada (UMNG) seja o mais apropriado e prático para construir um conhecimento duradouro, que possa ser aplicado. Neste trabalho, analisamos os resultados da implantação de tecnologias da informação e da comunicação no ensino-aprendizagem dos conhecimentos que o clínico geral deve ter quanto a patologias vasculares, em especial, as mais frequentes na prática clínica. Trata-se de um modelo de sala de aula virtual com módulos em que são apresentados os temas do currículo atual da matéria de Patologias Vasculares e Angiologia do programa de Medicina da UMNG, que segue o modelo de sala de aula invertida (flipped classroom). Entre os resultados, destaca-se uma melhora significativa das notas nos estudantes avaliados após cursarem o módulo virtual, tanto nos casos em que já tinham realizado o rodízio no serviço de cirurgia vascular quanto nos quais não tinham feito. A avaliação desse teste-piloto foi feita com vistas a determinar a efetividade de implantar, no futuro, um módulo virtual aplicável a maior escala na formação básica do clínico geral na faculdade de Medicina da UMNG.

Palavras-chave: aprendizagem; construção do conhecimento; ensino; flipped classroom; módulo virtual de aprendizagem; tecnologias da informação e da comunicação; patologia vascular 


\section{Introducción}

El método de enseñanza-aprendizaje utilizado actualmente con los estudiantes de pregrado de medicina está basado en la profundización de las áreas básicas como: medicina interna, cirugía general, ortopedia, pediatría y gineco-obstetricia, con lo cual se busca un mejor desempeño como médico general. Sin embargo, existen áreas, como la cirugía vascular, donde la frecuencia de las patologías ha incrementado a través de los años; aunque se les dedica poco tiempo en el periodo de formación de la carrera. En la Universidad Militar Nueva Granada (UMNG), por ejemplo, se le dedican dos semanas de rotación, que no son suficientes para satisfacer las necesidades de la población. Por lo anterior, se ha visto con preocupación el deficiente abordaje diagnóstico y terapéutico que muchos médicos generales realizan a los pacientes con patología vascular, lo que ha llevado a preguntarse por el origen de esta falencia y si el problema radica en las bases académicas durante el pregrado.

El pregrado de medicina de la UMNG consta de dos partes: la primera corresponde al abordaje de las ciencias básicas que se desarrollan del primero al quinto semestre; la segunda, la clínica, tiene lugar a partir del sexto semestre. Durante la parte clínica, los estudiantes pasan por las diferentes especialidades. Particularmente, en el servicio de Cirugía Vascular, los estudiantes de séptimo semestre rotan durante dos semanas incompletas, tiempo durante el cual realizan actividades tanto académicas como asistenciales. Adicionalmente, asisten a cuatro clases magistrales dictadas por cirujanos del área, en las que se abarcan las patologías más importantes que deben conocer y aprender a manejar como médicos generales.

Hace aproximadamente diez años, los estudiantes de medicina de séptimo semestre rotaban por el servicio de Cirugía Vascular durante cuatro semanas, asistiendo a todas las actividades del servicio, con cada uno de los especialistas. Durante ese tiempo, tenían la oportunidad de aprender de forma práctica y teórica las bases necesarias para el abordaje diagnóstico y terapéutico que un médico general necesita. Sin embargo, con el paso de los años, este lapso se ha visto reducido a apenas cuatro días a la semana, por lo que al final del semestre tan solo reciben seis horas de clases magistrales programadas para abarcar toda la patología vascular.

La evaluación de los estudiantes se realiza por medio de una prueba escrita al final de cada rotación. Las rotaciones son de cinco o seis estudiantes cada una, lo que implica que, al llegar a las clases teóricas, $90 \%$ de los estudiantes ya han rotado por el servicio y, por ende, ya han sido evaluados. Esta modalidad deriva en dos consecuencias: por un lado, que al rotar los estudiantes aún no tienen las bases para hacerlo y, por otro, que al finalizar el semestre ya han adquirido los conocimientos en el tema como producto de la rotación, por lo que las clases magistrales como tal no tienen una funcionalidad académica, ni tendrían un valor representativo en la evaluación. Esta situación, además genera desinterés en los estudiantes, ya que para el momento de las clases magistrales los estudiantes ya han aprobado el semestre.

Se considera que el tiempo que los estudiantes de medicina están en el área de cirugía vascular es poco para cubrir todos los temas requeridos. Además, en la actualidad no es posible cambiar ni modificar dichos tiempos, debido a que el currículo vigente está diseñado para que el estudiante aprenda el manejo de las patologías más comunes. No obstante, con el paso del tiempo las patologías vasculares han tomado auge y su frecuencia ha aumentado, lo que implica que el médico general deba estar capacitado en su abordaje.

Lo anterior ha llevado a cuestionarse sobre cuál es la estrategia educativa más precisa para ayudar a profundizar en los temas requeridos en cirugía vascular, para que el médico general esté más capacitado y, en consecuencia, disminuyan los errores diagnósticos y terapéuticos.

Por tal razón, el presente trabajo busca dar las herramientas para implementar una nueva estrategia educativa de enseñanza-aprendizaje, con base en un enfoque desde las Tic y en el método flipped classroom (aula invertida), dirigido a los estudiantes de séptimo semestre de medicina de la UMNG en el área de cirugía vascular y angiología. A su vez, este método es comparado con el tradicional de aprendizaje y evaluación, actualmente en uso. 
En definitiva, la estrategia propuesta tiene como fin permitir que los estudiantes adquieran los conocimientos básicos necesarios para el desempeño profesional como médicos generales, frente a los pacientes con patologías vasculares.

\section{Aportes de las tecnologías de la información y la comunicación}

Existen diferentes investigaciones en las que se han utilizado TIC como herramientas de trabajo, con miras a satisfacer las necesidades de todos los estudiantes, promoviendo nuevos métodos de aprendizaje. En el 2005, en Santiago de Chile, Chile, se implementó el software gratuito Sueñaletras, con el objetivo de apoyar a docentes en el proceso de enseñanza de lectoescritura a niños sordos e hipoacúsicos, con el que se demuestran los efectos benéficos globales de la alfabetización. Asimismo, en Bolivia, a través de una institución sin ánimo de lucro, se implementó el uso de Tic en el aula, en un programa llamado "Cerrando brechas", que permitió fortalecer las habilidades tecnológicas a más de 4000 docentes y 50000 alumnos, procurando el empoderamiento y desarrollo local. En Colombia, la universidad EA FIT desarrolló un laboratorio para la innovación del aprendizaje llamado "Proyecto 50", para potenciar las competencias de los docentes a través de la innovación de procesos de enseñanza, aprendizaje e investigación creativa con el uso de TIC. La fundación TR@Ms de Barcelona, España, implementó en el 2000 un trabajo colaborativo entre diferentes centros, usando recursos como tabletas, robots, portátiles e Internet, con el que se logró potenciar el uso de los recursos didácticos en un trabajo colaborativo interinstitucional (1-4).

En Ecuador el programa "Las distancias ya no son un obstáculo para la alfabetización digital" fue un programa que buscaba llevar la alfabetización a zonas rurales y urbanas marginadas, a través del Ministerio de Telecomunicaciones de ese país. Entre los resultado de esta iniciativa se cuentan cerca de 800000 ciudadanos alfabetizados con escuelas móviles (5).

Programas similares se han desarrollado en Argentina, Venezuela, Brasil y El Salvador, a fin de optimizar la enseñanza y el aprendizaje a través de plataformas digitales, llevando la educación a los más desfavorecidos; así, se ha profundizado la atención en los ámbitos social y educativo, con recursos tecnológicos a través de programas como "Educar", "Akdemia", "Ensanche" y "Para ti" (6-9).

Además de la iniciativa mencionada, en Colombia, podemos describir varios estudios y trabajos con respecto a las TIC, como el de Parra-Remolina, titulado "Las TIC como herramientas de reposicionamiento de la imagen corporativa. Caso Fundación Vascular", un trabajo en la Universidad Pontificia Bolivariana, de Bucaramanga, con el que se buscó el posicionamiento competitivo y con liderazgo de la institución. En 2013, en el sitio web Consultor Salud, Mejía-Medina presentó el trabajo "Impacto de las TIC en el sector salud", en el que mostró que el uso de las tecnologías, redes sociales, teléfonos inteligentes y correos electrónicos, etc., genera cada vez mayor impacto en todos los campos, incluyendo, el sector salud $(10,11)$.

Por otra parte, luego de evaluar las preferencias del uso de las Tic por los médicos residentes en los diferentes servicios de cirugía general, en Paraguay, Sandoval mostró que, en su mayoría, estos médicos están conectados con las tecnologías por diferentes métodos, con impacto positivos en su educación. Asimismo, Agámez-Luengas y su equipo publicaron un artículo que muestra cómo a nivel mundial se introduce en los currículos de medicina el uso libre de las tecnologías (asignaturas relacionadas con ciber- y telemedicina), que permite a los estudiantes un entrenamiento adecuado para saber buscar, retirar y usar datos necesarios; con lo cual mejora el cuidado de los pacientes $(12,13)$.

También se encontraron dos trabajos de la UMNG referentes al uso de TIC. Por un lado, el estudio de Mora-Guevara muestra que el desarrollo de tales herramientas brinda técnicas que permiten desarrollar ciber-competencias; esto, a su vez, facilita actividades desarrolladas por los individuos dentro de un contexto laboral o académico. Por otro, el estudio de Chica mostró que el uso de los ambientes virtuales de aprendizaje (AVA) permiten al docente ser tanto tutor como orientador en el proceso educativo, con flexibilidad, dinamismo, 
creatividad e inclusión. Esto revela que estas posibilidades tecnológicas han cambiado la manera de concebir, planificar y ejecutar los procesos de aprendizaje, así como la forma de percibir, acceder y transmitir el conocimiento $(14,15)$.

Estos son algunos antecedentes del avance de las TIC a nivel mundial y nacional, recursos tecnológicos que pueden ser una alternativa de enseñanza-aprendizaje para ser implementados en el área de cirugía vascular. Se plantea como alternativa para obtener un aprendizaje más efectivo y duradero, mientras se busca satisfacer las necesidades presentadas, con miras a disminuir las falencias que en la actualidad presentan los médicos generales y promover, con ello, la adecuada formación de estos profesionales de la salud.

Ahor bien, Jonathan Bergmann y Aarón Sams, en Colorado, EE. UU., fueron los primeros en experimentar el flipped classroom como motivación para los estudiantes. Esta iniciativa fue exitosa y sus resultados se han dado a conocer desde el 2015 por el New Media Consortium. En esa medida, Estados Unidos es pionero sobre los pronósticos de inserción en las Tic. En 2006-2007, se construyeron los primeros modelos del flipped classroom y, en 2008, se inició su implementación con propuestas más elaboradas; además, desde 2009, la implementación del modelo maestría invertida ha generado un proceso educativo con mayor autonomía del estudiante en la gestión de su formación (16).

\section{Objetivos}

El presente trabajo pretende buscar un nuevo paradigma educativo, diferente al actual, para la enseñanza-aprendizaje de los conocimientos de los estudiantes de pregrado (séptimo semestre), relacionados con el área de cirugía vascular y angiología de medicina, de la UMNG. El propósito, por tanto, es que al final de su carrera dichos estudiantes puedan tener los conocimientos necesarios que el médico general requiere para la atención adecuada, oportuna, humana y ética de los pacientes con patologías vasculares.

\section{Fundamentación teórica}

La discusión sobre los diferentes modelos educativos ya ha sido abordada desde la Antigüedad. Sócrates, (470-399 a. C.) sostenía que aprender es recordar y que el conocimiento está en cada ser humano. El método de aprendizaje por descubrimiento, propuesto en la dialéctica socrática, sostenía que el conocimiento debía ser adquirido por el esfuerzo propio del estudiante (17).

Asimismo, Platón (427-347 a. C.) planteó que la educación debía empezar a temprana edad y sin diferenciación de clase social o género, por lo que propuso el juego como método de aprendizaje. También creó la Academia, donde se enseñaba, a través del diálogo; a su vez, motivado por la libertad y en un sentido sociocultural, sostenía: "No hay ninguna disciplina que deba aprender el hombre libre por medio de la esclavitud. El alma no conserva ningún conocimiento que haya penetrado por la fuerza" (18).

Por su parte, Confucio (551-479 a. C) habló de la importancia estratégica de la práctica social como fuente primaria del conocimiento. Señaló: “Me lo contaron y lo olvidé, lo vi y lo entendí, lo hice y lo aprendí" (19). De estos preceptos, se consideró el valor del aprendizaje a partir de la interacción de los sentidos con el medio social, haciendo parte de lo que se entiende como la unión de la cabeza, el corazón y la mano.

Dando un salto en el tiempo, nos encontramos con Rousseau (1712-1778), quien consideró que el educando es el principal protagonista del proceso de aprendizaje y debe tener plena libertad para hacer lo que quiera, ya que, al final, terminará haciendo lo que debe. Así, la idea es dar vía libre a la experiencia autónoma, para que el estudiante descubra por sí mismo, de modo que este método heurístico constituya la clave de la educación. A su turno, Vygotsky (1896-1934), considerado el padre del constructivismo social, propone que el conocimiento es producto de la interacción social y cultural. Para él y Piaget, las teorías de la actividad del desarrollo constituyen los pilares más recientes del aprender haciendo. Del mismo modo, Dewey (1859-1952), pionero en las indagaciones sobre el campo experimental de la modernidad, destacó el 
carácter interactivo en la construcción de saberes y defendió la idea de un aprendizaje orientado a la acción a partir del proyecto escuela activa (19).

A pesar de que la lista de personajes importantes en esa materia es extensa, la idea de este recuento es mostrar que las nuevas tendencias educativas ya han sido analizadas por muchos de ellos y que el flipped classroom ha estado implícito desde el principio. Así que lo que puede hacer la diferencia hoy en día, en el campo de la enseñanza y el aprendizaje, es el uso de las tecnologías en los campos hacia los que los diferentes filósofos, psicólogos, científicos, médicos y pensadores de la historia se han enfocado, lo que ha llevado a un cambio en los modelos educativos, pensados para el futuro social y cultural de los alumnos.

Las tecnologías y la comunicación son un punto relevante en la educación actual y, como dijo Dewey, si enseñamos a los estudiantes como pensábamos en el pasado, les estamos robando el futuro. Así, la innovación juega un papel importante en los procesos educativos, ya que tiene que responder a una exigencia del presente y aportar una solución real de los problemas educativos. La innovación es el medio por el cual la sociedad se mantiene vigente y más en la educación, donde el profesor, el estudiante y, ahora, también la tecnología son los principales actores en la formación educativa (20).

En la era de la información y con los diferentes proyectos educativos, el aporte fundamental de los profesores es en la renovación y reinvención de las metodologías, centradas en la enseñanza para el aprendizaje, en las que el estudiante es el centro, a la vez que la educación y la innovación son dinámicas. Esta práctica implica aprender, desaprender y estar preparados para la innovación (21).

En cuanto herramienta, la creatividad es parte fundamental del aprendizaje, pues ayuda a que los estudiantes hagan parte activa del proceso formador, con lo cual el rendimiento y la satisfacción por los contenidos de los programas docentes mejora; al mismo tiempo que la deserción escolar se reduce y se incrementa la transferencia en el lugar de trabajo. Según Bernstein, el entretenimiento se convierte en una estrategia creativa, persuasiva y fundamental para cualquier tipo de comunicación, que supone activar mecanismos cognitivos, que nos permitan sentirnos atraídos (20).

Tavárez-Rodríguez muestra que todos los aspectos de la vida y la actividad humana han sido modificados por el progreso tecnológico. Desde luego, la educación es una de ellas, centrada en el estudiante. Por esto, el maestro de hoy no puede estar alejado de las tecnologías y, por ende, debe disponer de ellas en el aula de clase. Existe gran variedad de reformas educativas con miras a la competitividad y ofrecer a los ciudadanos mejores condiciones de vida; además, el docente tiene la responsabilidad de los cambios en los procesos educativos y del aprendizaje, enfocado en las herramientas tecnológicas y cómo aplicarlas en el aula de clase (21).

El aula invertida es una modalidad que genera más tiempo en clase, trabajo en equipo, respuestas a preguntas y trabajo con proyectos. A través de este modelo, el docente se dedica a resolver preguntas, siendo un facilitador de los proyectos asignados, ya que graba videos para ser vistos posteriormente; para desarrollar los proyectos en clase, dotando a los alumnos de autonomía en su proceso de aprendizaje; volteando el foco de atención, alejándolo del profesor para dirigirlo hacia el estudiante. A partir de 2014, se creó la flipped learning network (FLN), para promover en los educadores conocimientos y habilidades para implementar el aula invertida. Los cuatro principios básicos del flipped classroom son:

- Ambiente flexible que facilita la interacción y reflexión del aprendizaje.

- Cultura de aprendizaje que genera el protagonismo de los estudiantes para adquirir conocimientos.

- El contenido intencional, que genera la prioridad de los conceptos relevantes para el estudiante.

- El educador profesional que da el apoyo permanente para adquirir conocimiento de una forma práctica y responsable.

El flipped classroom promueve al docente como facilitador y al estudiante como creador del conocimiento. Así las cosas, el nuevo paradigma es el siguiente: el $70 \%$ se aprende a través del desarrollo 
del trabajo y experiencia; un $20 \%$ se adquiere a través de evaluaciones y conciencia personal; y el $10 \%$ restante hace parte de la formación dirigida por un instructor, por lo que el enfoque actual es el de aprender haciendo (22).

La frase de Benjamin Franklin: "Dime y lo olvido, enséñame y lo recuerdo, involúcrame y lo aprendo" (23) nos introduce en el reto de los contenidos, en los que las tecnologías digitales y las herramientas interactivas han transformado la educación dentro y fuera del aula, aportando formas nuevas y participativas de observar la realidad y aprender de ella. Por esta razón, las innovaciones tecnológicas tienen impacto en los contenidos educativos y sobre las nuevas posibilidades en el mundo de la educación. Así, el reto en los contenidos y, desde el punto de vista educativo, no pasa por el deslumbramiento tecnológico, sino por saber aprovechar y adaptar la tecnología, para llevar contenidos de calidad a un estadio superior, bien por su capacidad motivadora, su fomento del aprendizaje colaborativo, o bien por su apuesta por el cambio social (23).

En su momento, Paulo Freire dijo que "enseñar no es transferir conocimiento", sino "crear la posibilidad de producirlo". Por eso, la misión de los educadores es crear las condiciones necesarias para que los estudiantes aprendan; deben elegir en cada momento qué metodologías utilizar, refinándolas y adaptándolas según las necesidades propias de cada aprendizaje. Las actividades que deben diseñarse pueden desarrollarse en diversos planos sociales, individuales o en grupo; involucrando al alumno de forma pasiva o activa; llevando desde la adquisición hasta el descubrimiento, el debate, la creación o la práctica y teniendo en cuenta que no hay una metodología más efectiva que otras, sino que depende de cada situación educativa (23).

Según Cabrera Rayo, en estos tiempos en los que la profesionalización de la enseñanza es buscada por universidades y médicos interesados en aprender a enseñar, se vuelve indispensable buscar, encontrar y aplicar organizadamente los métodos de enseñanza. Asimismo, se considera que, desde Hipócrates y hasta la actualidad, ningún método didáctico aislado debe considerarse ideal. Si se abordan las necesidades grupales e individuales y la profesionalización de la docencia, no puede desvincularse una herramienta didáctica del resto; por tanto, la combinación de todas las herramientas, tanto del aula como las tecnológicas, favorecen el aprendizaje de forma significativa. Si a esto se suma una imagen de respeto, responsabilidad y humanismo que el médico debe irradiar y contagiar a sus alumnos, se formarán no solo médicos comprometidos con la sociedad y consigo mismos, sino además excelentes seres humanos (24).

\section{Materiales y métodos}

A fin de implementar una estrategia educativa en la enseñanza-aprendizaje en el área de cirugía vascular y angiología, como alternativa para cambiar el paradigma educativo, se desarrolló una prueba piloto en estudiantes de séptimo semestre de medicina de la UMNG, con la cual se buscaba optimizar el proceso de aprendizaje. Se implementó la estrategia educativa flipped classroom, para la enseñanza, aprendizaje y evaluación de los estudiantes de séptimo semestre de medicina. Y se comparó con el actual método de aprendizaje y evaluación con el método nuevo propuesto en este trabajo; ofreciendo a los estudiantes una construcción del conocimiento firme para ser puesta en práctica en un futuro como médicos generales.

Se realizó un módulo virtual como prueba piloto que incluyó: presentación en PowerPoint de los temas específicos en el área de cirugía vascular; revisión de las guías del servicio de cirugía vascular del Hospital Militar Central; un texto base para ampliación de la literatura; vínculos a artículos de revisión y con la página de la Asociación Colombiana de Cirugía Vascular y Angiología y un formato de autoevaluación virtual de los temas revisados.

Los docentes fueron tutores, mediadores y acompañantes activos del aprendizaje. Así, en cada rotación, los estudiantes adquirieron las bases teóricas y pusieron en práctica los conocimientos adquiridos, con la asesoría de los docentes y especialistas del servicio durante la rotación, buscando, con este método, un aprendizaje más activo y con conocimientos duraderos (flipped classroom). 
El módulo o curso de cirugía vascular se dejó instalado en la página web de la UMNG, específicamente en el aula virtual, disponible solo para los estudiantes de medicina de séptimo semestre, del período en cuestión, donde debían inscribirse y, posteriormente, revisar el contenido. El módulo permaneció disponible desde las 08:00 horas del 23 de octubre hasta las 00:00 horas del 2 de noviembre del 2018.

Al final del módulo, los estudiantes presentaron un examen virtual y, posteriormente, una encuesta de evaluación referente a este, con lo cual se finalizaron los objetivos del curso. Antes de iniciar el módulo, se realizó una prueba escrita de los mismos temas que estaban en el módulo y que hacían parte del currículo actual, prueba realizada el 23 de octubre a las 06:00 horas.

A partir del 3 de noviembre, se realizó una comparación y un análisis de los resultados obtenidos en ambas pruebas y de la encuesta de evaluación del módulo. A partir de los resultados de las diferentes evaluaciones, se definió la pertinencia de la implementación del nuevo método, realizando una propuesta formal con metas a la implementación de los módulos y del curso, modificando el currículo operativo en el área de cirugía vascular.

\section{Resultados}

El martes 23 de octubre del 2018, cuarenta y nueve $(\mathrm{N}=49)$ estudiantes de séptimo semestre de Medicina de la UMNG, presentaron una prueba escrita basada en los temas básicos de cirugía vascular que un médico general debe saber en su práctica diaria y que actualmente hacen parte del currículo del semestre en esta materia.

Se establecieron dos grupos: el grupo 1, conformado por 35 estudiantes que ya habían pasado por la rotación de dos semanas en el servicio de Cirugía Vascular del Hospital Militar Central; y el grupo 2, con 14 estudiantes que aún no habían rotado por el servicio.

Los resultados obtenidos fueron: en el grupo 1, el $34,28 \%$ de los estudiantes obtuvo una nota inferior a $3,5(\mathrm{n}=12)$ y el $65,72 \%$ obtuvo una nota por encima de 3,5; del grupo 2 se encontró que el $57,14 \%(\mathrm{n}=8)$ obtuvo una nota por debajo de 3,5 , mientras que el $42,8 \%$ restante $(n=6)$ obtuvo una nota mayor a 3,5 .

Del total de los 49 estudiantes, 10 obtuvieron nota por debajo de 3,0 (20\%); 20 tuvieron nota inferior a 3,5 (40,8\%); y 29, por encima de ese valor $(59,2 \%)$, tal como se muestra en las tablas 1 y 2.

Tabla 1. Promedios por grupos del pre-test

\begin{tabular}{|c|c|c|c|}
\hline \multicolumn{1}{|c|}{ Descripción } & $\begin{array}{c}\text { Nota } \\
\text { mínima }\end{array}$ & $\begin{array}{c}\text { Nota } \\
\text { máxima }\end{array}$ & Promedio \\
\hline Grupo 1 $(n=35)$ & 2,25 & 4,75 & 3,58 \\
\hline Grupo 2 $(n=14)$ & 1,75 & 4,0 & 3,09 \\
\hline Total $(N=49)$ & 1,75 & 4,75 & 3,44 \\
\hline
\end{tabular}

Tabla 2. Rango de calificaciones del pre-test

\begin{tabular}{|c|c|c|c|}
\hline \multicolumn{1}{|c}{ Nota } & Grupo 1 & Grupo 2 & Total \\
\hline Menos de 2,0 & 0 & $1(7,1 \%)$ & $1(2,04 \%)$ \\
\hline $2,0-2,5$ & $1(2,8 \%)$ & $1(7,1 \%)$ & $2(4,08 \%)$ \\
\hline $2,5-3,0$ & $4(11,4 \%)$ & $3(21,4 \%)$ & $7(14,2 \%)$ \\
\hline $3,0-3,5$ & $7(20 \%)$ & $3(21,4 \%)$ & $10(20,4 \%)$ \\
\hline $3,5-4,0$ & $9(25,7 \%)$ & $4(28,5 \%)$ & $13(26,5 \%)$ \\
\hline $4,0-4,5$ & $12(34,2 \%)$ & $2(14 \%)$ & $14(28,5 \%)$ \\
\hline Más de 4,5 & $2(5,7 \%)$ & 0 & $2(4,08 \%)$ \\
\hline
\end{tabular}

Basados en el nuevo modelo propuesto ( $f l i$ pped classroom) el mismo 23 de octubre, se abrió el curso virtual de cirugía vascular en la plataforma Moodle del aula virtual de la UMNG, con los mismos temas exigidos en el programa y dividido en los siguientes módulos: introducción, semiología, insuficiencia venosa, enfermedad arterial, enfermedad carotídea, aneurisma de aorta y trauma vascular; junto con un módulo de examen (test) y un módulo de autoevaluación. Para llevar a cabo las actividades del curso, se dieron dos semanas como plazo (mismas que el estudiante rota por el servicio de Cirugía Vascular), para ser estudiado y evaluado dentro de la misma plataforma. La fecha límite para que fuera completado fue el 3 de noviembre del 2018, hasta las 00:59 horas. 
Los grupos estaban conformados de la siguiente manera: grupo 1, compuesto por 29 estudiantes que ya habían pasado por la rotación de dos semanas en el servicio de Cirugía Vascular del Hospital Militar Central. Por su parte, el grupo 2 estuvo configurado con 10 estudiantes que aún no habían rotado.

Los resultados fueron del siguiente orden: del grupo 1 , faltaron cinco estudiantes que no realizaron el curso ni presentaron la prueba; del grupo 2, fueron cuatro los ausentes. Asimismo, del grupo 1, se encontró que el $13,33 \%$ tuvo una nota inferior a $3,5(n=4)$, mientras que el $86,67 \%$ restante obtuvo una nota por encima de 3,5. Del grupo 2, se encontró que el $30 \%(n=3)$ obtuvo una nota por debajo de 3,5 , y el $70 \%$ restante $(n=7)$ obtuvo una nota mayor a 3,5 .

Por otro lado, del total de los 40 estudiantes, cuatro no superaron la nota $(13,3 \%)$; siete tuvieron nota inferior a $3,5(24,13 \%)$ y veintidós estudiantes estuvieron por encima de ese valor $(75,86 \%)$, tal como se expone en las tablas 3 y 4 .

Tabla 3. Promedios por grupo de la evaluación luego del módulo virtual

\begin{tabular}{|c|c|c|c|}
\hline \multicolumn{2}{|c}{ Descripción } & $\begin{array}{c}\text { Nota } \\
\text { mínima }\end{array}$ & $\begin{array}{c}\text { Nota } \\
\text { máxima }\end{array}$ \\
\hline Grupo 1 $(n=29)$ & 1,87 & 4,79 & 3,84 \\
\hline Grupo 2 $(n=10)$ & 1,25 & 4,58 & 3,64 \\
\hline Total $(N=39)$ & 1,25 & 4,79 & 3,81 \\
\hline
\end{tabular}

Tabla 4. Rango de la evaluación luego del módulo virtual

\begin{tabular}{|c|c|c|c|}
\hline \multicolumn{1}{|c}{ Nota } & Grupo 1 & Grupo 2 & Total \\
\hline Menos de 2,0 & $1(3,4 \%)$ & $1(10 \%)$ & $2(5,12 \%)$ \\
\hline $2,0-2,5$ & 0 & $1(10 \%)$ & $1(2,56 \%)$ \\
\hline $2,5-3,0$ & $1(3,4 \%)$ & 0 & $1(2,56 \%)$ \\
\hline $3,0-3,5$ & $2(6,9 \%)$ & $1(10 \%)$ & $3(7,7 \%)$ \\
\hline $3,5-4,0$ & $15(51,72 \%)$ & $3(30 \%)$ & $18(46,1 \%)$ \\
\hline $4,0-4,5$ & $5(17,2 \%)$ & $2(20 \%)$ & $7(18 \%)$ \\
\hline Más de 4,5 & $5(17,2 \%)$ & $2(20 \%)$ & $7(18 \%)$ \\
\hline
\end{tabular}

Para comparar los promedios de las notas de los dos grupos tanto antes como después de estudiar el módulo virtual, se aplicó una prueba $t$ de estudiante; adicionalmente, se compararon los resultados del total de estudiantes (grupo 1 más grupo 2), antes y después del módulo. Se encontraron diferencias significativas en los puntajes en el grupo 1 y en la totalidad de los estudiantes antes y después; y se evidenciaron mayores diferencias con valores $\mathrm{p}$ significativos: $\mathrm{p}=0,01$ para la comparación del total de estudiantes antes y después; $y$ $\mathrm{p}=0,04$ para el grupo 1 antes y después. Aunque no hubo diferencias significativas al comparar el grupo 2. Finalmente, se logró identificar un tendencia hacia la satisfacción en el grupo 2 después del módulo, pero no alcanzó a ser significativa, debido al tamaño de la muestra.

Se dejó un módulo de evaluación del curso virtual para ser realizado por los estudiantes y responder las preguntas, luego de finalizado. Para ello, se realizaron seis preguntas sobre si el curso sirvió de apoyo para el proceso actual de aprendizaje; si el curso mejoró la adquisición de conocimientos para futuro como médico general; si, comparado con el método actual, este curso sería una mejor herramienta educativa; si los estudiantes están de acuerdo con la implementación del nuevo curso para ser aplicado, y si estarían de acuerdo con aplicarlo en otras áreas de la medicina. Adicionalmente, se dejó una pregunta abierta para sugerencias. Las respuestas a las anteriores preguntas de evaluación arrojaron lo siguiente: el 78,57\% de los estudiantes estuvo total o parcialmente de acuerdo, mientras que el $21,43 \%$ no lo estuvo.

En cuanto a sugerencias, el factor común de las respuestas fue dejar el módulo desde el inicio del semestre; aplicarlo durante las rotaciones de los estudiantes por el servicio de Cirugía Vascular y tener la oportunidad de revisarlo con la actividad asistencial, para poder correlacionarlo con la parte práctica. Con este último módulo, se dio por finalizado el curso virtual de cirugía vascular dentro del aula virtual de la UMNG. 


\section{Conclusión}

Los procesos educativos de enseñanza-aprendizaje han venido cambiando desde hace muchos años, tanto a nivel mundial como nacional, con enfoques que permiten una educación integral. Dichos cambios se han presentado en todas las áreas y la medicina no es ajena a ellos.

Las herramientas tecnológicas son de gran ayuda para que la construcción del conocimiento para el alumno sea agradable, de interés y duradera. Por tanto, los docentes deben estar preparados para aprender y desaprender, con el fin de ser facilitadores del conocimiento también a través del uso de TIC.

A pesar de ser un estudio piloto, el trabajo presentado demostró buenos resultados. Las pruebas presentadas mostraron una mejoría significativa en las notas con el curso virtual, tanto en los estudiantes que habían pasado por el área de cirugía vascular, como en aquellos que no.

El desarrollo del módulo virtual se presentará para ser evaluado en comité de currículo con miras a ser implementado y adicionado, así como para que sea objeto de sugerencias por parte de profesores y alumnos, para darle un desarrollo adecuado, que permita diseñar un mejor método de enseñanza.

Es necesario seguir trabajando con proyección hacia el futuro profesional de los estudiantes, con el ánimo de conseguir mejores profesionales, con sentido humano, ético y de la responsabilidad; en el campo de la medicina, debe pensarse en el óptimo desempeño de los médicos generales, con la seguridad y calidad que los pacientes requieren.

\section{Referencias}

1. Sauvalle I. Aprendizaje integrador: Proyecto de Sueñaletras [Internet]. 2005 [consultado el 20 de noviembre de 2019]. Disponible en http://www.cedeti.cl/software-educativo/sueñaletras/

2. Ortega-Azurdy S. Cerrando brechas [Internet]. Bolivia. 1998 [consultado el 20 de noviembre de 2019]. Disponible en http://redayni.org

3. Zea-Restrepo CM. Laboratorio para la innovacion y aprendizaje [Internet]. Medellín; 2010 [consultado el 20 de noviembre de 2019]. Disponible en http://www. eafit.edu.co/proyecto50/
4. Romero Am. Ipads, robots e internet invaden la escuela [Internet]. Trams fundación; 2000 [consultado el 20 de noviembre de 2019]. Disponible en http://www.fundaciotrams.org/

5. Guerrero-Ruiz J. Las distancias ya no son un obstáculo para la alfabetización digital en Ecuador [Internet]. Ecuador; 2011 [Consultado el 20 de noviembre de 2019]. Disponible en http://telecomunicaciones.gob. ec/

6. Pomies P. Cuando el Aula se Convierte en Ordenador [Internet]. Argentina; 2000 [consultado el 20 de noviembre de 2019]. Disponible en http://www.educ.ar/

7. Muñoz E. Akdemia, una mejor educación en tiempo real [Internet]. 2011 [consultado el 20 de noviembre de 2019]. Disponible en https://www.akdemia.com/

8. Alvarenga RE. Ensanchando las TIC para educar a la comunidad [Internet]. El Salvador; 2009 [consultado el 19 de noviembre de 2019]. Disponible en http://ensanche.gob

9. Urani L. Una Educación con TIC desde las favelas [Internet]. Brasil; 1990 [consultado el 18 de noviembre de 2019]. Disponible en https://www.parationg.org/en

10. Remolina P, Carolina S. Las TIC como herramientas de reposicionamiento de la imagen corporativa. Caso Fundación Vascular [Internet]. Bucaramanga: Universidad Pontificia Bolivariana; 2013 jun [citado el 20 de diciembre de 2019]. Pp. 98. Disponible en https://repository.upb.edu.co/handle/20.500.11912/280

11. Mejía-Medina F, ConsultorSalud. Impacto de las TIC en el Sector Salud [Internet]. ConsultorSalud. 2014 [citado el 20 de diciembre de 2019]. Disponible en https:// consultorsalud.com/impacto-de-las-tic-en-el-sectorsalud/

12. Sandoval J. Acceso, uso y preferencias de las TIC por médicos residentes de cirugía general en el Paraguay. Cir Parag. 2017; 41(2):8-12. Doi:10.18004/sopaci.agosto.08-12.

13. Agamez-Luengas S, Aldana-Bolaño $M$, Barreto-Ar$\cos$ V, Santana-Goenaga A, Caballero-Uribe CV. Aplicación de nuevas tecnologías de la información en al enseñanza de la medicina. Salud Uninorte, 2009; 25(1):150-72.

14. Mora Guevara A. Implementación de las Tic en educación médica [Internet] [especialización]. [Bogotá]: Universidad Militar Nueva Granada; 2013 [citado el 20 de diciembre de 2019]. Disponible en http://repository. unimilitar.edu.co/handle/10654/12994

15. Chica MP. Ambientes virtuales de aprendizaje (AVA) como herramientas tecnológicas educativas y su impacto en la educación superior en Iberoamérica [Internet] [Especialización]. [Bogotá]: Universidad Militar 
Nueva Granada; 2018 [citado el 20 de diciembre de 2019]. Disponible en: http://repository.unimilitar.edu. co/handle/10654/17840

16 Trujillo GR. Del learning by doing al learning by living, aprender haciendo: la educacion que mira hacia el futuro. En: Pérez-Tornero JM, Tejedor S, editores. Innovación Educativa y Tics: Guía Básica. Barcelona, España: Cooperativa Editorial Magisterio, Editorial UOC; 2017. Pp. 171-85.

17. Reibelo-Martín J. Método de enseñanza-aprendizaje para la enseñanza por descubrimiento. Aula Abierta. 1998; 71:123-47.

18. Nieto-Blanco C, Abascal-Cobo. Lecturas de historia de la filosofia [Internet]. Santander, Cantabria, España: Publican Ediciones, Universidad de Cantabria; 1996.

19. Becerra-Plaza Ge. Innovación. En: Pérez-Tornero JM, Tejedor S (eds.). Innovación Educativa y TIC: guía básica. Barcelona, España: Cooperativa Editorial Magisterio, Editorial Uoc; 2017. Pp. 41-47.
20. Bernstein B. Class, codes and control: the structuring of pedagogic discourse. Londres: Routledge; 1990.

21. Tavarez-Rodríguez JA. Docente 3.0 en el entorno iberoamericano. En: Pérez-Tornero JM, Tejedor S (eds.). Innovación educativa y TIC: guía básica. Barcelona, España: Cooperativa Editorial Magisterio, Editorial Uoc; 2017. Pp. 63-71.

22. Peralta-García L. Los Contenidos. En Pérez-Tornero JM, Tejedor S (eds.). Innovación educativa y TIC: guía básica. Barcelona, España: Cooperativa Editorial Magisterio, Editorial uoc; 2017. Pp. 125-135.

23. Davinia HL. Las metodologías. En Pérez-Tornero JM, Tejedor S (eds.). Innovación educativa y TIC: gtuía básica. Barcelona, España: Cooperativa Editorial Magisterio, Editorial uoc; 2017. Pp. 153-9.

24. Cabrera-Rayo A. Buscando el metodo de aprendizaje ideal. De Hipócrates al siglo xxi. Arch Med Urg Méx. 2012;4(2):49-50. 